\title{
dspace.vutbr.cz
}

\section{Applications of novel behavioral implementation of a controllable generalized current conveyor}

ŠOTNER, R.; LANGHAMMER, L.; PETRŽELA, J.; DOMANSKÝ, 0.; DOSTÁL, T.

Proceedings of the 2018 28th International Conference Radioelektronika (RADIOELEKTRONIKA)

pp. 1-6

elSBN: 978-1-5386-2485-2

DOl: http://dx.doi.org/10.1109/RADIOELEK.2018.8376350

Accepted manuscript

C2018 IEEE. Personal use of this material is permitted. Permission from IEEE must be obtained for all other uses, in any current or future media, including reprinting/republishing this material for advertising or promotional purposes, creating new collective works, for resale or redistribution to servers or lists, or reuse of any copyrighted component of this work in other works. Roman Sotner, Lukas Langhammer, Jiri Petrzela, Ondrej Domansky, Tomas Dostal, "Applications of novel behavioral implementation of a controllable generalized current conveyor", Proceedings of the 2018 28th International Conference Radioelektronika (RADIOELEKTRONIKA), pp. 1-6, 2018. DOI: 10.1109/RADIOELEK.2018.8376350. Final version is available at https://ieeexplore.ieee.org/abstract/document/8376350/ 


\section{Applications of Novel Behavioral Implementation of a Controllable Generalized Current Conveyor}

\author{
Roman Sotner, Lukas Langhammer, Jiri Petrzela, \\ Ondrej Domansky \\ SIX Research Center, Department of Radio Electronics \\ Brno University of Technology \\ Brno, Czech Republic \\ sotner@feec.vutbr.cz
}

\author{
Tomas Dostal \\ Dept. of Technical Studies \\ College of Polytechnics \\ Jihlava, Czech Republic \\ tomas.dostal@vspj.cz
}

\begin{abstract}
This contribution presents a novel modification of a behavioral model for a generalized current conveyor of second generation based on commercially available active devices. This model is suitable for preliminary tests of applications before demanding stage of expensive IC design and fabrication starts. Its main features aim at simplifying and unifying topology where three independently electronically adjustable parameters are available. Operation of the model is verified in several introduced applications (an electronically reconfigurable filter and generation of sine waveform). Substantial extension of tunability has been obtained in applying the model in the proposed oscillator in comparison to the standard linear tuning method. All expectations were confirmed by PSpice simulations.
\end{abstract}

Keywords- Behavioral model; current conveyor; electronic control; extended tuning; multi-parameter control

\section{INTRODUCTION}

Current conveyors (CCs) [1], [2] are important active elements for many various building blocks and other active subparts [3]. Their importance in applications increased with the development of controllable features. It started with the study of implementing adjustable current gain $(B)$ between $X$ and $\mathrm{Z}$ terminal $\left(I_{\mathrm{Z}}=B I_{\mathrm{X}}\right)$ of the three-port current conveyor of second generation (CCII) [4]-[6]. Then, other ways of control were tried, for example adjustability of the input "parasitic" resistance $\left(R_{\mathrm{X}}\right)$ of terminal $\mathrm{X}\left(V_{\mathrm{X}}=R_{\mathrm{X}} I_{\mathrm{X}}\right)$ [7]. However, there are significant problems with large-signal behavior and linearity. The next milestone was obtained by combining several controllable parameters within the frame of the device. An interesting attempt was provided by designing a conveyor allowing current gain $B$ as well as voltage gain $(A)$ to be adjusted between $\mathrm{Y}$ and $\mathrm{X}$ terminals $\left(V_{\mathrm{X}}=A V_{\mathrm{Y}}\right)$ [8]. The idea of bringing together adjustability of $R_{\mathrm{X}}$ and $B$ [9] is very important because it significantly improves the degree of freedom in many applications (independent controllability of pole frequency and quality factor in biquad filters, independent driving conditions for oscillation and frequency of oscillators, etc.) using one or two of these active devices. All discussed definitions were verified and prepared as bipolar or unipolar internal topologies for possible IC design. However, for common designers and practicians these so-called behavioral models of active devices are important in order to verify design

Research described in this paper was financed by Czech Ministry of Education in frame of National Sustainability Program under grant LO1401. For research, infrastructure of the SIX Center was used. intentions before expensive IC fabrication.

Recent literature introduced several models of active devices related to the discussed topic. Methods of intentional $R_{\mathrm{X}}$ control were a topic for several works [10]-[12]. The functionality of $R_{\mathrm{X}}$ control was confirmed but some concepts have limited usability (only current followers or amplifiers with controllable $R_{\mathrm{X}}$ and gain $B$ ) [10], [12] or their complexity is a substantial drawback [11]. Solutions in [10] and [12] cannot be used as full three-port current conveyors. The solution proposed in this contribution uses the approach introduced in [12] and extends a current amplifier (two-port) to a three-port current conveyor. It significantly reduces the complexity of solutions presented in [11]. All of these results are compared in tabular form (Tab. I) with key works [4]-[9] and with a new proposal.

Organization of this paper is as follows: Section II brings a short overview of a generalized type of current conveyor and its transformation to a generalized current conveyor of second generation, a survey of important recent works in this field and specification of their deficiencies. Section III introduces a new concept of a behavioral model and Section IV provides several application examples. Typical performances are summarized in Section V. Verification of selected applications is presented in Section VI, Section VII explains the detailed relation with our own previous works and Section VIII concludes this work.

\section{STATE-OF-THE-ART}

The hybrid matrix equation of a generalized three-port current conveyor (GCC) [13], [14] has the form:

$$
\left[\begin{array}{l}
V_{X} \\
I_{Y} \\
I_{Z}
\end{array}\right]=\left[\begin{array}{lll}
0 & \alpha & 0 \\
\beta & 0 & 0 \\
\gamma & 0 & 0
\end{array}\right] \cdot\left[\begin{array}{c}
I_{X} \\
V_{Y} \\
V_{Z}
\end{array}\right] .
$$

In recent decades, the second-generation type of $\mathrm{CC}$ (abbreviated as CCII) [1]-[3] was developed, where parameters of (1) are fixed and defined as: $\alpha=1, \beta=0$ and $\gamma=1$. The widespread usage of this CCII type confirms its usefulness for various applications. However, the lack of adjustability of the CCII parameters results in limited controllability. Only values of external passive elements can be used for the purpose of 
tuning. Many modifications of CCII (in order to adjust their parameters) have been introduced in recent years (the most important are compared in Tab. I).

The concept of CCII with adjustable inter-terminal transfers and input resistance of $\mathrm{X}$ terminal is called the generalized current conveyor of second generation (GCCII) [11]. The following system of equations defines GCCII behavior as:

$$
\left[\begin{array}{l}
V_{X} \\
I_{Y} \\
I_{Z}
\end{array}\right]=\left[\begin{array}{ccc}
R_{X} & A & 0 \\
0 & 0 & 0 \\
B & 0 & 0
\end{array}\right] \cdot\left[\begin{array}{c}
I_{X} \\
V_{Y} \\
V_{Z}
\end{array}\right],
$$

where $R_{\mathrm{X}}$ represents adjustable input resistance of terminal X, $A$ adjustable voltage transfer between $\mathrm{Y}$ and $\mathrm{X}$ (open) terminal and $B$ adjustable current transfer between $\mathrm{X}$ and $\mathrm{Z}$ terminal.

Motivation of this work can be explained as an attempt to improve the version of a previously presented GCCII [11] model combined with a beneficial and simplified method of $R_{\mathrm{X}}$ control introduced in [12]. The complexity of the solution presented in [11] leads to searching for simpler topology with similar controllable features. In addition, implementation of GCCII in [11] consists of a higher number of different types of active elements and a high number of passive elements. Unfortunately, the method of $R_{\mathrm{X}}$ control presented in [12] is not a fully implementable form for current conveyor modeling (only a two-port current amplifier/follower is solved in [12] and [10]). In addition, the approach presented in [10] can be easily replaced by the concept shown in [12] with better performance and lower overall complexity. An advanced active element having multi-parameter controllability results in significantly extended tunability and adjustability of applications [12], [15].

TABLE I. COMPARISON OF CONTROLlable CCIIs, DERIVED MODELS AND MODIFICATIONS

\begin{tabular}{|l|c|c|c|c|c|c|}
\hline & & & & \\
& & &
\end{tabular}

\section{PROPOSED SOLUTION}

Complexity of the solution [11], where many different types of active elements (voltage buffers, variable gain amplifiers, diamond transistors) as well as the number of passive elements (two or three external resistors) which are required, and the missing terminal $\mathrm{Y}$ in concept [10], [12] (solution presented as current follower or amplifier with controllable features) motivate our work and presented results.

Figure 1 introduces the topology of the newly proposed behavioral model of GCCII. The topology consists of an electronically controllable CCII (ECCII) whose purpose is to set current gain $(B)$ by DC control voltage $V_{\text {SET_B. }}$ ECCII in topology can be replaced by a simpler type of device (CCII as available in OPA860 [16], AD844 [17], etc.) if adjustable $B$ is not necessary in some cases $(B=1)$. The rest of the circuit uses an interconnection of variable gain voltage amplifiers (VGAs) controlled by driving voltages $V_{\text {SET_RX }}\left(R_{\mathrm{X}}\right.$ control $)$ and $V_{\mathrm{SET} \text { A }}$ (A control) and a differential voltage buffer (DVB). This differential buffer can also be replaced by VGA with its gain set to $A=1$. Then, the majority of active devices in the topology is the same type in comparison to [11]. The floating resistor $R_{\mathrm{x}_{-} \text {int }}$ (hundreds of $\Omega$ recommended for high frequency applications) creates a very important part of topology that serves for $R_{\mathrm{X}}$ definition and value control by using the bootstrap technique. VGAs are represented by AD835 [18] multipliers connected in suitable arrangement as shown in [15]. In addition, implementation of a voltage-mode multiplier brings an additional feature to the proposed concept. Voltage gain $A$ can reach both polarities very simply (change of $V_{\text {SET_A }}$ polarity). This can be useful in specific applications. ECCII was solved by EL2082 [19] and a current inverter connected at the $\mathrm{Z}$ terminal (when required) based on the diamond transistor OPA860 [16]. Despite the obsolete status of EL2082, the device is still being distributed and its operation can be easily replaced by the solution shown in [11]. Parameters of GCCII in Fig. 1 can be expressed as:

$$
R_{X}=\frac{R_{x_{-} \text {int }}}{1+A_{R x}} \cong \frac{R_{x_{-} \text {int }}}{1+4 V_{S E T_{-} R x}},
$$

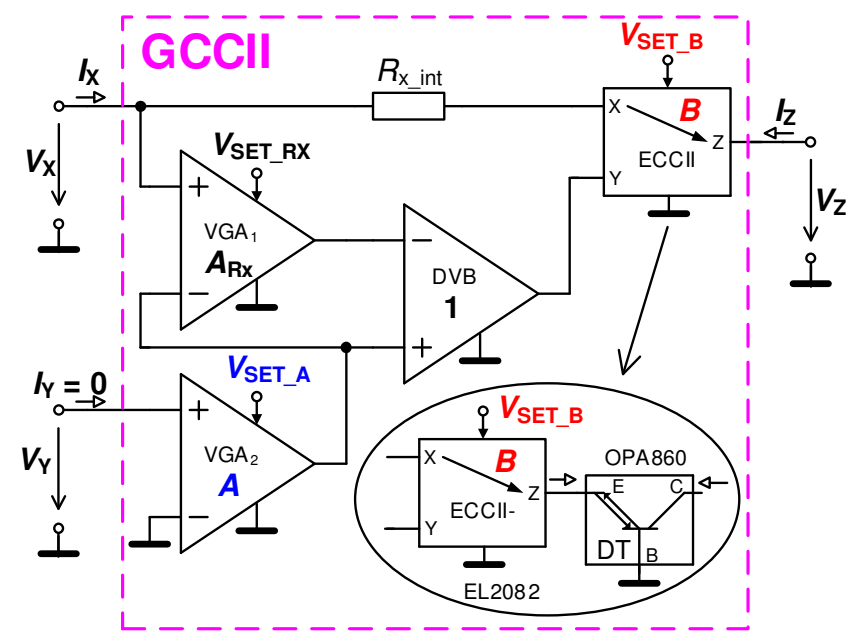

Fig. 1. Newly proposed behavioral model of GCCII. 


$$
\begin{aligned}
V_{X} \cong A V_{Y}-\left(\frac{R_{x_{-} \text {int }}}{1+4 V_{S E T_{-} R x}}\right) I_{X}, \\
A \cong 4 V_{S E T_{-} A}, \\
I_{Z}=B I_{X} \cong V_{S E T_{-} B} I_{X} .
\end{aligned}
$$

Note that the form (4) is valid when current $I_{X}$ flows (load at $X$ terminal) in the opposite direction than shown in Fig. 1 (twoport definition). Otherwise (open $\mathrm{X}$ ), $V_{\mathrm{X}} \cong A V_{\mathrm{Y}}$.

\section{APPLICATION EXAMPLES}

GCCII offers various implementations in many analog signal-processing applications. The following examples target utilization of selected or all available parameters adjustable within the GCCII model in Fig. 1.

\section{A. Reconfigurable lossless/lossy voltage-mode integrator}

GCCII and the low number of external passive elements offer an interesting type of voltage-mode integrator (Fig. 2) that has an electronically tunable time constant (by parameters $B$ and $R_{\mathrm{X}}$ ) as we can see from the following transfer relation:

$$
K(s)=\frac{\frac{B}{R_{X} C}}{s+\frac{A B}{R_{X} C}},
$$

therefore, extending integrator adjustability is also possible as well as reconfiguring the integrator between lossy and lossless operation selectable by the value of gain $A(A=0$ easily available when voltage mode multiplier forms $\mathrm{VGA}_{2}$ in the GCCII topology in Fig. 1). The designation GCCII- means inverted current of the output terminal $\mathrm{Z}$ in comparison to the two-port definition (Fig. 1).

\section{B. Reconfigurable first-order filter}

The topology of a filtering application is shown in Fig. 3. Again, implementation of a multiplier in the position of $\mathrm{VGA}_{2}$ brings interesting features for reconfigurability. As we can see, $R_{\mathrm{X}}$ serves for tuning the filter and $A$ and $B$ or both are able to reconfigure the general transfer function:

$$
K(s)=\frac{s+\frac{C_{2} \pm A B C_{1}}{R_{X} C_{1} C_{2}}}{s+\frac{1}{R_{X} C_{1} C_{2}}},
$$

into inverting the all-pass filter (iAP) for $A=-2(B=1)$ or high-pass filter (HP) for $A=-1 \quad(B=1)$ and supposing $C_{1}=C_{2}=C$. We can save controllable parameter $A$ (reduction of complexity of GCCII in Fig. $1, \mathrm{VGA}_{2}$ can be omitted) because the same operation is obtained for $A=1$ (always fixed) and $B=-1$ or -2 . It requires only changing the polarity of the current from the $\mathrm{Z}$ terminal of GCCII.

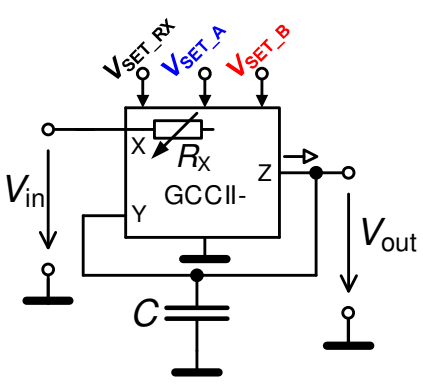

Fig. 2. Reconfigurable first-order lossy/lossless integrator with extended tunability.

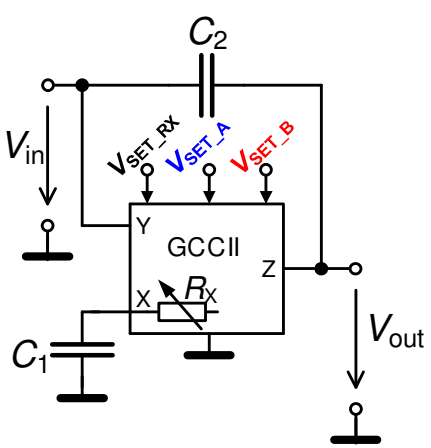

Fig. 3. Reconfigurable first-order filter.

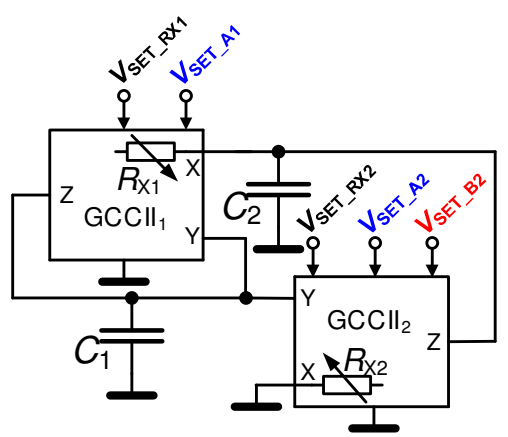

Fig. 4. Oscillator with extended tunability.

\section{Oscillator with extended tunability}

The last example of a GCCII application focuses on the design of electronically adjustable oscillators. Two GCCIIs are implemented in very simple topology illustrated in Fig. 4. The characteristic equation of the oscillator has the form:

$$
s^{2}+\frac{C_{1}-C_{2} A_{1} B_{1}}{R_{X 1} C_{1} C_{2}} s+\frac{B_{1} B_{2} A_{2}}{R_{X 1} R_{X 2} C_{1} C_{2}}=0 .
$$

The voltage gain $A_{1} \geq C_{1} / C_{2}\left(B_{1}=1\right)$ ensures controllability of the condition for oscillation. It means $A_{1} \geq 1$ for equal values of capacities $\left(C_{1}=C_{2}=C\right)$. Parameters $R_{\mathrm{X} 1}, R_{\mathrm{X} 2}, A_{2}, B_{2}$ should be used for controlling the frequency of oscillation (FO).

1) Linear tuning of frequency

Simultaneous control of $R_{\mathrm{X} 1}=R_{\mathrm{X} 2}=R_{\mathrm{X}} \quad$ (supposing $A_{2}=B_{2}=1$ ) allows linear tuning of FO in accordance to: 


$$
\omega_{0}=\frac{1}{R_{X} C} \cong \frac{1+4 V_{S E T_{-} R x}}{R_{x_{-} \mathrm{int}} C} .
$$

This arrangement leads to the following relation between produced voltage signals across $C_{1}$ and $C_{2}$ as:

$$
\frac{V_{C 2}}{V_{C 1}}=\left.\frac{2}{1+s C R_{X}}\right|_{s=j \omega_{0}} \Rightarrow \frac{V_{C 2}}{V_{C 1}}=1-j=\sqrt{2} \exp \left(-j \frac{\pi}{4}\right) .
$$

The oscillator produces an unchangeable level of output signals with constant ratio $\sqrt{ } 2$ and constant phase shift $45^{\circ}$ when FO is tuned linearly.

\section{2) Extended control of frequency}

The type of active devices used for $A_{2}, B_{2}$ and $R_{\mathrm{X}}$ control (linear dependence of parameters on DC voltage) ensures simple simultaneous control of parameters for driving FO. Replacement of gains by control voltages for all three parameters results in the expression for FO:

$$
\omega_{0}=\frac{A_{2} B_{2}}{R_{X} C} \cong \frac{2 \sqrt{V_{S E T_{-} A 2} V_{S E T_{-} B 2}}\left(1+4 V_{S E T_{-} R X}\right)}{R_{x_{-} \mathrm{int}} C} .
$$

Simultaneous control of FO by a single voltage $\left(V_{\mathrm{SET} \_\mathrm{A} 2}=V_{\mathrm{SET} \_\mathrm{B} 2}=V_{\mathrm{SET} \_\mathrm{RX}}=V_{\mathrm{SET}}\right)$ requires modification of the control voltage for $A_{2}\left(V_{\mathrm{SET} \_\mathrm{A} 2}=V_{\mathrm{SET}} / 4\right)$ [15] in order to ensure that gain values $\left(A_{2}\right.$ and $\left.B_{2}\right)$ are identical, then (12) leads to:

$$
\omega_{0} \cong \frac{V_{S E T}\left(1+4 V_{S E T}\right)}{R_{x_{-} \mathrm{int}} C} \cong \frac{4 V_{S E T}^{2}+V_{S E T}}{R_{x_{-} \mathrm{int}} C} .
$$

Unfortunately, independence of output levels and phase distance of produced signals is now disrupted by the tuning process by $V_{\mathrm{SET}}$ :

$$
\begin{aligned}
& \frac{V_{C 2}}{V_{C 1}}=\frac{A_{2} B_{2}+1}{1+s C R_{X}} \cong \frac{V_{S E T}^{2}+1}{1+j V_{S E T}} \Rightarrow \\
& \Rightarrow \frac{V_{C 2}}{V_{C 1}}=\sqrt{1+V_{S E T}^{2}} \exp \left[\tan ^{-1}\left(-V_{S E T}\right)\right]
\end{aligned} .
$$

However, it cannot be considered as a significant drawback if the single-phase output of the oscillator is sufficient for the application. One amplitude remains constant during the tuning of FO (a correctly applied system for amplitude control and stabilization expected) whereas variation of the second level is not rapid in dependence of $V_{\text {SET }}$ from 0.1 to $1 \mathrm{~V}$.

\section{TyPICAL PERFORMANCE OF THE GCCII MODEL}

Simulation results of the important transfer AC and DC properties as well as impedance plot of terminal $\mathrm{X}$ are introduced in the following text. Important results are summarized in Tab. II and examples of $X$ terminal impedance characteristics are shown in Fig. 5. The expected ideal ranges of parameter values are: $B=0 \rightarrow 1[-], A=0 \rightarrow 4[-]$ and $R_{\mathrm{X}}=1000 \rightarrow 200 \Omega$ (all for $V_{\text {SET_(A/B/RX) }}=0 \rightarrow 1 \mathrm{~V}$ ). The simulated results are in good agreement with expectations.
TABLE II. BAsic Performances of The Model (Fig. 1)

\begin{tabular}{|c|c|c|c|}
\hline parameter & $B$ & $A$ & $R_{\mathrm{X}}$ \\
\hline$V_{\mathrm{SET} \_/ / B / R \mathrm{x}}$ & $0 \rightarrow 1 \mathrm{~V}$ & $0 \rightarrow 1 \mathrm{~V}$ & $0 \rightarrow 1 \mathrm{~V}$ \\
\hline DC value & $0 \rightarrow 1[-]$ & $0 \rightarrow 3.8[-]$ & $1000 \rightarrow 212 \Omega$ \\
\hline dynamics $^{\mathrm{a}}$ & $> \pm 1.4 \mathrm{~mA}$ & $> \pm 0.8 \mathrm{~V}$ & $> \pm 1.2 \mathrm{~mA}$ \\
\hline bandwidth & $64 \rightarrow 102 \mathrm{MHz}$ & $16 \rightarrow 48 \mathrm{MHz}$ & $>8 \mathrm{MHz}$ \\
\hline
\end{tabular}

${ }^{a}$ good linearity without limitation (tested in all combinations of parameters) a)

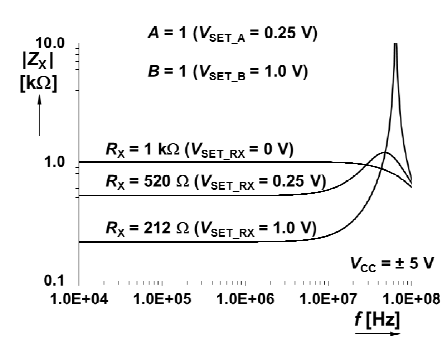

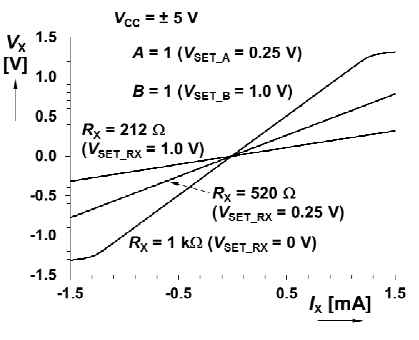

b)
Fig. 5. Exemplary results for AC and DC analysis of GCCII (Fig. 1): a) magnitude impedance vs frequency plot (terminal X), b) DC response at X.

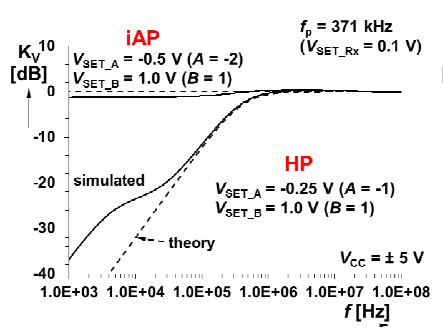

a)

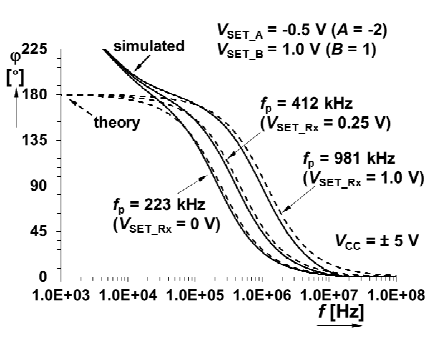

b)
Fig. 6. Frequency responses of reconfigurable filter: a) magnitude responses for iAP and HP transfer, b) phase responses for iAP and pole frequency tuned.

\section{VERIFICATION OF SELECTED APPLICATIONS}

Selected GCCII applications were designed and simulated in PSpice (version 16.6) and results (compared with theoretical expectations) are given in the following subsections.

\section{A. Reconfigurable first-order filter}

This circuitry (Fig. 3) was designed for the following parameters: $C_{1}=C_{2}=C=680 \mathrm{pF}, \quad R_{\mathrm{x} \_ \text {int }}=1 \mathrm{k} \Omega$ and pole frequency $f_{\mathrm{p}}=400 \mathrm{kHz}$. Thus, the remaining parameter can be calculated (8) as $R_{\mathrm{X}}=585 \Omega$ and $V_{\text {SET_RX }} \cong 0.18 \mathrm{~V}$ based on (3). Figure 6(a) compares ideal and simulated results for operating of the filter as HP or iAP for different values of $A=-$ 1 and $-2\left(V_{\text {SET }_{-} \mathrm{A}}=-0.25\right.$ and $\left.-0.5 \mathrm{~V}\right)$. Figure $6(\mathrm{~b})$ shows tuning of the $\mathrm{iAP}$ responses for $V_{\mathrm{SET} \_\mathrm{RX}}=0,0.25$ and $1 \mathrm{~V}$. Ideal values of pole frequencies are found as 234, 468 and $1170 \mathrm{kHz}$. Simulated results follow these expectations as indicated in Fig. 6(b). Real features of active elements in the simulated behavioral model bring issues with parasitic zeros and poles in the stop band (HP). These effects especially relate with real parasitic capacitance and resistance of the "internal" X terminal of the used ECCII device in the model.

\section{B. Oscillator with extended tunability}

Analysis of the topology from Fig. 4 was performed in time domain for identical values of capacitors and $R_{\mathrm{x} \_ \text {int }}$ as in 
previous analyses. The oscillator was complemented by the system for amplitude stabilization based on a diode-doubler rectifier and operational-amplifier-based amplifier serving as a subtracter of the DC reference level and error level from the rectifier. The rectifier input was supplied from the $C_{2}\left(V_{\mathrm{C} 2}\right)$ node after impedance separation (buffer). Parameter $A_{1}$ $\left(\mathrm{GCCII}_{1}\right)$ was driven $\left(V_{\mathrm{SET}} \mathrm{A} \mathrm{A}\right)$ by the produced DC voltage of the opamp. This circuit was tested in two cases. The first case focuses on linear tuning of $\mathrm{FO}$ by $R_{\mathrm{X} 1,2}$ (simultaneously $R_{\mathrm{X} 1}=R_{\mathrm{X} 2}=R_{\mathrm{X}}$ ) parameters of both GCCIIs (whereas $B_{1}=$ $B_{2}=A_{2}=1$ ) that results in unchangeable output levels and constant phase angle (distance) between $V_{\mathrm{C} 1}$ and $V_{\mathrm{C} 2}$ theoretically equal to $45^{\circ}$. Control voltage $V_{\text {SET_RX }}$ was changed from 0.1 up to $1 \mathrm{~V}$ and ideal FO values expected between 328 and $1171 \mathrm{kHz}$. Simulations provided a tunability range from 308 up to $1033 \mathrm{kHz}$. High nodal parasitic capacitances (sum of terminal capacities reaches more than $10 \mathrm{pF}$ in node of $C_{1}$ ) cause major influence on lower values of a simulated frequency. Transient responses for $V_{\text {SET_RX }}=0.25$ and $1 \mathrm{~V}$ are shown in Fig. 7. The ratio of produced amplitudes clearly confirms the validity of (11) as well as the simulated phase shift which was evaluated as $45^{\circ}$ and $46^{\circ}$ for waveforms in Fig. 7. The value of total harmonic distortion (THD) was calculated close to $1 \%$ or lower in the observed range and in dependence on the specific setting of system for amplitude stabilization.

The second case of operation of the proposed oscillator takes into account all suitable parameters for tuning of FO $\left(R_{\mathrm{X} 1}, R_{\mathrm{X} 2}, B_{2}, A_{2}\right)$ that are driven by the single DC control voltage with simplified notation $V_{\text {SET }}$. This simultaneous control of all discussed parameters is possible because DC voltage serving to adjust $A_{2}$ (based on multiplier AD835) is divided by 4 (resistor divider) and then in accordance to (5) and (6) VGAs (AD835) and ECCII (EL2082) are driven by the same voltage. Both devices reach the same values of gain ( $\left.B_{2}=A_{2} \cong V_{\mathrm{SET}}\right)$ in the ideal case. The oscillator has been tested by simulations in this mode of operation for the same range of $V_{\text {SET }}$ values as in the previous case $(0.1 \rightarrow 1 \mathrm{~V})$. Expected minimal and maximal FO from ideal theory reaches $33 \mathrm{kHz}$ and $1171 \mathrm{kHz}$. Simulations yield $21 \mathrm{kHz} \rightarrow 999 \mathrm{kHz}$. It means a really significant extension of $\mathrm{FO}$ readjustability. The resulting waveforms are shown in Fig. 8 again for $V_{\mathrm{SET}}=0.25$ and $1 \mathrm{~V}$. Impact of the tuning process on generated amplitudes is visible but not drastic in accordance to (14) and values of $V_{\text {SET }}$. Unfortunately, phase shift $16^{\circ}$ and $47^{\circ}$ has been obtained for the lowest and the highest frequency shown in Fig. 8 as expected. That makes this operational mode suitable only for widely tunable single phase generation. THD was obtained in similar values as in the previous case, however, units of percent were obtained for the changed output level $\left(V_{\mathrm{C} 1}\right)$ when approaching saturation of active devices. Precise setting of the system for amplitude stabilization is always responsible for THD and fluctuation of levels. Unfortunately, it is impossible to reach optimal setting in long-running transient simulations.

Figure 9 compares both methods of FO tuning. The first way offers theoretical retunability $\Delta f_{0}=843 \mathrm{kHz}$ (ratio $\left.f_{0 \max } / f_{0 \min }=3.6: 1\right)$ while the second way $\Delta f_{0}=1138 \mathrm{kHz}$ $\left(f_{0 \max } / f_{0 \min }=36: 1\right)$. Simulations yield $f_{0}=712 \mathrm{kHz} \quad$ (ratio $f_{0 \max } / f_{0 \min }=3.3: 1$ ) in the first case and $f_{0}=978 \mathrm{kHz}$ (ratio $\left.f_{0 \max } / f_{0 \min }=48: 1\right)$ in the second case.

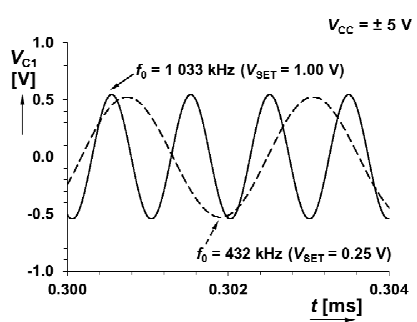

a)

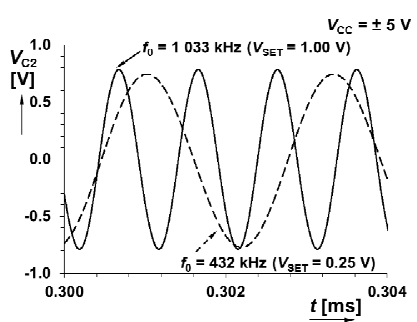

b)
Fig. 7. Output waveforms in high-impedance nodes for two selected discrete values of $V_{\mathrm{SET} \_\mathrm{RX}}\left(\right.$ tuned by $R_{\mathrm{X} 1,2}$ only): a) $V_{\mathrm{C} 1}$, b) $V_{\mathrm{C} 2}$.

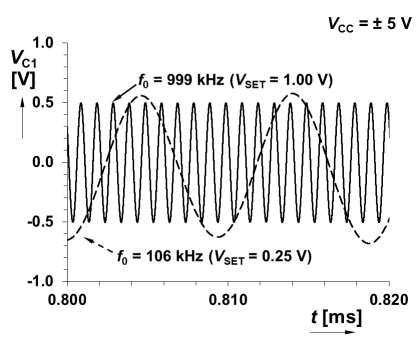

a)

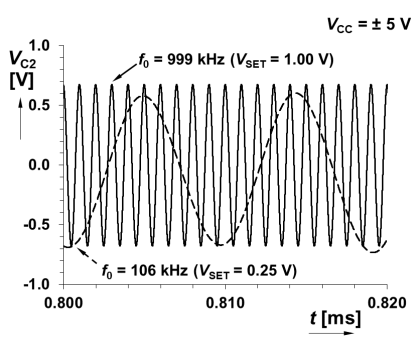

b)
Fig. 8. Output waveforms in high-impedance nodes for two selected discrete values of $V_{\mathrm{SET}}$ : (used all suitable parameters $\left.-R_{\mathrm{X} 1}, R_{\mathrm{X} 2}, B_{2}, A_{2}\right)$ a) $V_{\mathrm{C} 1}$, b) $V_{\mathrm{C} 2}$.

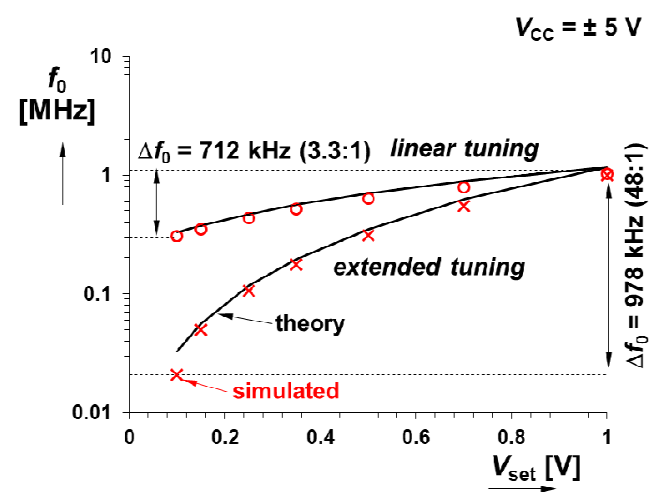

Fig. 9. Comparison of dependence of FO on $V_{\mathrm{SET}}$ for linear $\left(R_{\mathrm{X} 1,2}\right.$ only) and extended type of tuning $\left(R_{\mathrm{X} 1}, R_{\mathrm{X} 2}, B_{2}, A_{2}\right)$.

\section{DETAILED DisCUSSION OF PREVIOUS RECENT WORKS}

This paper presents a new model of a GCCII device. This model represents an extension of the topology of the adjustable current amplifier introduced in [12]. However, there are many topological and functional differences. First of all, circuitry of the model in [12] is not intended for purposes of GCCII operations. The work in [12] only discusses a simple adjustable current amplifier (single input and single or multiple outputs) utilizing single VGA, single ECCII and single resistor in the simplest form (single input single output type). The newly presented model in Fig. 1 requires two VGAs, DVB and ECCII in order to fulfill all requirements for a fully controllable GCCII device (an additional Y terminal and adjustable voltage gain $A$ between $\mathrm{Y}$ and $\mathrm{X}$ terminals that is not available in works [10] and [12]). Paper [10] only deals with topologies of 
current amplifiers (two-ports). However, these topologies are complicated in comparison to [12]. Moreover, old models in [10] focus on topologies employing only ECCIIs (three of these devices are required) and topologies employing two VGAs and a diamond transistor. The core of the circuit in Fig. 1 and cores of published topologies in [10] are completely different. The new concept of the GCCII model in this paper (Fig. 1) targets the design of a three-port device (current conveyor). This is a significant difference compared to previously presented works [10], [12] and this new paper. The two topologies in [11] create the first introduced behavioral models of GCCII (having a Y terminal and a relation between $\mathrm{Y}$ and $\mathrm{X}$ terminals - adjustable voltage gain $A$ ). However, both concepts in [11] are complex (solution 1: two voltage buffers, two ECCIIs, VGA and two resistors; solution 2: two VGAs, diamond transistor, differential buffer, ECCII and three resistors). The solution in Fig. 1 tends to simpler circuitry (two VGAs, differential buffer, ECCII and single resistor) than models in [11] (one active device and a majority of resistors are saved) and, therefore, it brings significant improvement in comparison to [11]. Therefore, the circuitry in Fig. 1 is original and it solves some drawbacks of [11] as well as extends controllable inter-terminal features (parameters) of previously reported current amplifiers [10], [12] where inspiration was found also for this work. From a practical point of view, the model in Fig. 1 surpasses the possibilities ( $\mathrm{Y}$ terminal, operation $V_{\mathrm{X}}=V_{\mathrm{Y}} \cdot A$ for open $\mathrm{X}$ ) of solutions from [10] and [12] and simplifies the resulting circuitry in comparison to the old version of GCCII [11].

In addition, no application examples of mentioned GCCIIs are introduced in works [10] and [11]. The content of this paper also explains and analyzes three application examples (two of them in detail) of the proposed GCCII from Fig. 1. Note that these applications (special integrator, reconfigurable filter and oscillator in Fig. 2, Fig. 3 and Fig. 4) are not synthesizable by the concepts from [10] and [12] due to missing Y terminal and the relation between $\mathrm{Y}$ and $\mathrm{X}$ terminals.

\section{VIII.CONCLUSION}

Results regarding new applications of the generalized current conveyor of second generation were presented in this paper. The contribution of extended controllability of GCCII parameters leads to interesting applications (reconfigurable lossy/lossless integrator, reconfigurable first-order filter and sine wave oscillator). Especially the last application confirms the benefits of multi-parameter control of GCCII devices by significantly extending the tunability of the generated frequency (more than 10-times in comparison to the linear way of FO tuning). These features were obtained by significantly improving and modifying the model of GCCII compared to the presented one in previous paper [11].

\section{REFERENCES}

[1] A. Sedra, K. C. Smith, "A second generation current conveyor and its applications," IEEE Transaction on Circuit Theory, vol. CT-17, no. 2, pp. $132-134,1970$.
[2] J. A. Svoboda, L. McGory, S. Webb, "Applications of a commercially available current conveyor," Int. Journal of Electronics, vol. 70, no. 1, pp. 159-164, 1991.

[3] D. Biolek, R. Senani, V. Biolkova, Z. Kolka, "Active elements for analog signal processing: Classification, Review and New Proposals," Radioengineering, vol. 17, no. 4, pp. 15-32, 2008.

[4] W. Surakampontorn, W. Thitimajshima, "Integrable electronically tunable current conveyors," IEE Proceedings-G, vol. 135, no. 2, pp. 7177, 1988.

[5] A. Fabre, N. Mimeche, "Class A/AB second-generation current conveyor with controlled current gain," Electronics Letters, vol. 30, no. 16, pp. 1267-1268, 1994.

[6] S. Minaei, O. K. Sayin, H. Kuntman, "A new CMOS electronically tunable current conveyor and its application to current-mode filters," IEEE Transactions on Circuits and Systems - I, vol. 53, no. 7, pp. 14481457, 2006.

[7] A. Fabre, O. Saaid, F. Wiest, C. Boucheron, "High frequency applications based on a new current controlled conveyor," IEEE Transactions on Circuits and Systems - I, vol. 43, no. 2, pp. 82-91, 1996.

[8] A. Marcellis, G. Ferri, N. C. Guerrini, G. Scotti, V. Stornelli, A. Trifiletti, "The VGC-CCII: a novel building block and its application to capacitance multiplication," Analog Integrated Circuits and Signal Processing, vol. 58, no. 1, pp. 55-59, 2009.

[9] M. Kumngern, S. Junnapiya, "A sinusoidal oscillator using translinear current conveyors," in Proc. Asia Pacific Conf. on Circuits and Systems APPCAS2010, Kuala Lumpur, 2010, pp. 740-743

[10] R. Sotner, A. Kartci, J. Jerabek, N. Herencsar, T. Dostal, K. Vrba, “An Additional Approach to Model Current Followers and Amplifiers with Electronically Controllable Parameters from Commercially Available ICs," Measurement Science Review, vol. 12, no. 6, pp. 255-265, 2012.

[11] J. Jerabek, R. Sotner, A. Kartci, N. Herencsar, T. Dostal, K. Vrba, "Two Behavioral Models of the Electronically Controlled Generalized Current Conveyor of the Second Generation," in Proc. 38 $8^{\text {th }}$ Int. Conf. on Telecommunications and Signal Processing TSP2015, Prague, 2015, pp. 349-353.

[12] R. Sotner, N. Herencsar, J. Jerabek, L. Langhammer, J. Polak, "On practical construction of electronically controllable compact current amplifier based on commercially available elements and its application," AEU - International Journal of Electronics and Communications, vol. 81, no. 11, pp. 56-66, 2017.

[13] J. Cajka, T. Dostal, K. Vrba, "General view on current conveyors," International Journal of Circuit Theory and Applications, vol. 32, no. 3, pp. 133-138, 2004.

[14] R. Senani, D. R. Bhaskar, A. K. Singh, Current Conveyors: Variants, Applications and Hardware Implementations, Springer, Berlin, Germany, 2015.

[15] R. Sotner, J. Jerabek, N. Herencsar, J. Petrzela, "Methods for Extended Tunability in Quadrature Oscillators Based on Enhanced Electronic Control of Time Constants," IEEE Transactions on Instrumentation and Measurement, in press, 2018.

[16] Texas Instruments. OPA860 Wide-bandwidth, operational transconductance amplifier (OTA) and buffer (datasheet), 2008, 33 p., accessible on www: http://www.ti.com/lit/ds/symlink/opa860.pdf

[17] Analog Devices. AD844 $60 \mathrm{MHz}, 2000 \mathrm{~V} / \mathrm{us}$ Monolithic Op Amp with Quad Low Noise (datasheet), 2017, 20 p., accessible on www: http://www.analog.com/media/en/technical-documentation/datasheets/AD844.pdf

[18] Analog Devices. AD835 $250 \mathrm{MHz}$, Voltage Output, 4-Quadrant Multiplier (datasheet), 2014, 15 p., accessible on www: http://www.analog.com/media/en/technical-documentation/datasheets/AD835.pdf

[19] Intersil (Elantec). EL2082 CN Current-mode multiplier (datasheet), 1996, 14 p., accessible on http://www.intersil.com/data/fn/fn7152.pdf 